INPLASY

PROTOCOL

To cite: Yuan et al. Healthrelated factors and all-cause mortality in non-hospitalized elderly population: an umbrella review of meta-analyses. Inplasy protocol 202130039. doi:

10.37766/inplasy2021.3.0039

Received: 13 March 2021

Published: 13 March 2021

Corresponding author:

Yin Yuan

13459475582@139.com

Author Affiliation:

Fujian Provincial Hospital, Fuzhou, Fujian Province, China

Support: FJMP 2019 Y9027.

Review Stage at time of this submission: Preliminary

searches.

Conflicts of interest:

None declared.

\section{Health-related factors and all-cause mortality in non-hospitalized elderly population: an umbrella review of meta-analyses}

Yuan, Y1; Huang, F²; Zhu, P³.

Review question / Objective: The aim of the present umbrella review is to synthesize the available evidence on healthrelated factors for all-cause mortality in non-hospitalized elderly population through a systematic review of metaanalyses, and assess the methodological quality and diverse biases in these meta-analyses, as well as grade the strength of evidence.

Condition being studied: Healthy aging is a major public health burden globally. The physical function, psychological spirit, social support and other aspects of comprehensive health status in elderly population are found to be associated with the occurrence and development of negative outcomes such as death, disability or hospitalization. With the publication of the results of the original cohort study, the evidence of observational meta-analysis on the factors related to the risk of death in the elderly population has gradually emerged. However, the epidemiological credibility of these meta-analyses has not been evaluated in the whole field.

INPLASY registration number: This protocol was registered with the International Platform of Registered Systematic Review and Meta-Analysis Protocols (INPLASY) on 13 March 2021 and was last updated on 13 March 2021 (registration number INPLASY202130039).

\section{INTRODUCTION}

Review question / Objective: The aim of the present umbrella review is to synthesize the available evidence on health-related factors for all-cause mortality in nonhospitalized elderly population through a systematic review of meta-analyses, and assess the methodological quality and diverse biases in these meta-analyses, as well as grade the strength of evidence.

Rationale: Plenty of meta-analyses of observational studies have indicated numerous factors for all-cause mortality in the elderly. However, the evidence for these associations has not been summarized, and the epidemiological credibility has not 
been appraised across the field. We plan to perform an umbrella review of the evidence across existing meta-analyses, aiming to summarize the influential factors associated with mortality in elderly population, and assess which of the previously studied associations have robust evidence.

Condition being studied: Healthy aging is a major public health burden globally. The physical function, psychological spirit, social support and other aspects of comprehensive health status in elderly population are found to be associated with the occurrence and development of negative outcomes such as death, disability or hospitalization. With the publication of the results of the original cohort study, the evidence of observational meta-analysis on the factors related to the risk of death in the elderly population has gradually emerged. However, the epidemiological credibility of these metaanalyses has not been evaluated in the whole field.

\section{METHODS}

Search strategy: We will systematically search electronic databases from 1st January 2000 to December 312020 to identify meta-analyses of observational longitudinal studies examining the association of influential factors with allcause mortality in older population. The search was limited to"English" language publications and human studies. Key words related to (1) health-related factors; (2) allcause mortality, death, survival, life expectancy, longevity, and (3) systematic review OR meta-analys*.

Participant or population: Elderly population (aged $\geq 60$ ) from community or nursing homes.

Intervention: Not applicable.

Comparator: Exposed group and nonexposed group.

Study designs to be included: Prospective or retrospective cohort studies.
Eligibility criteria: Selected studies met the following inclusion criteria: (a) metaanalyses in the elderly population (more than $90 \%$ of participants were aged $\geq 60$ years, or with a mean age over 60 years, or studies with mixed age groups, separately reporting data for the elderly), (b) metaanalyses of the longitudinal relationship between health-related factors with allcause mortality, (c) meta-analyses of cohort studies with more than six months follow-up duration. Studies were excluded if meta-analyses investigated selected populations, such as those with a specific diagnosis (e.g. hypertension, cancer), or meta-analyses evaluated the association with mortality with unchangeable factors such as age, sex, height, race, etc.

Information sources: The electronic databases including PubMed, Embase, Web of Science, Scopus and EBSCOhost were searched for relevant publications. Citations, reference list and reviews were manually searched for relevant studies.

Main outcome(s): All-cause mortality.

Quality assessment / Risk of bias analysis: Methodological quality of included metaanalyses was evaluated using the Assessing the Methodological Quality of Systematic Reviews (AMSTAR-2) tool, which consists of 16 items used to assess the quality of systematic reviews or metaanalysis. A rating system to rate and classify all reviews into critically low quality, low quality, moderate quality and high quality.

Strategy of data synthesis: The analysis will be performed in "Stata 15.0" software. For each meta-analysis, we will extract original data and estimate the summary effect size and its $95 \% \mathrm{Cl}$ using random-effects models. The random effects model is chosen as it represented a more conservative approach for studies with high heterogeneity. The $95 \%$ prediction interval (PI) will also be estimated. We will quantify heterogeneity between studies using the coefficient of inconsistency (12). We will assess small-study effects using the Egger's regression asymmetry test. We 
will perform the excess statistical significance test, which evaluates whether there is a relative excess of formally significant findings in the published literature due to any reason. Assessment of epidemiological credibility will be applied as well.

Subgroup analysis: If necessary, we will stratify the comparisons into several groups such as age, gender, or community/ nursing home, etc.

Sensitivity analysis: We will perform a sensitivity analysis limited to prospective cohort studies and retrospective cohort studies, respectively. We will examine whether the level of epidemiological credibility changes.

Language: English.

Country(ies) involved: China.

Keywords: Umbrella review, health-related factors, elderly, all-cause mortality.

Contributions of each author:

Author 1 - Yin Yuan.

Author 2 - Feng Huang.

Author 3 - Pengli Zhu. 